\title{
Perfect Entanglement Transport in Quantum Spin Chain Systems
}

\author{
Sujit Sarkar \\ PoornaPrajna Institute of Scientific Research, Bangalore, India \\ E-mail: sujit.tifr@gmail.com \\ Received June 20, 2011; revised November 24, 2011; accepted December 5, 2011
}

\begin{abstract}
We propose a mechanism for perfect entanglement transport in anti-ferromagnetic (AFM) quantum spin chain systems with modulated exchange coupling and also for the modulation of on-site magnetic field. We use the principle of adiabatic quantum pumping process for entanglement transfer in the spin chain systems. We achieve the perfect entanglement transfer over an arbitrarily long distance and a better entanglement transport for longer AFM spin chain system than for the ferromagnetic one. We explain analytically and physically - why the entanglement hops in alternate sites. We find the condition for blocking of entanglement transport even in the perfect pumping situation. Our analytical solution interconnects quantum many body physics and quantum information science.
\end{abstract}

Keywords: Quantum Many Body Models, Entanglement Physics, Quantum Antiferromagnetic Spin Chain Model, Adiabatic Transport

\section{Introduction}

Quantum communication between distant co-ordinates in a quantum network is an important requirement for quantum computation and information. One can construct the quantum network in different ways. Optical systems are typically employed in quantum communication and cryptography applications to transfer the state between two distinct co-ordinates directly via photons [1]. Quantum computing applications work with trapped atoms to transfer information between distant sites - photons in a cavity QED [2-6]. However, we would like to study the entanglement transfer through the quantum spin chain systems. The potentiality of the spin chain system - antiferromagnetic (AFM) and ferromagnetic (FM) - as a network of quantum state and entanglement transport has already been studied by many groups as referred in the literature [7-22]. The experimental evidence of nanoscale spin chain and their properties have been discussed in Ref. [23].

It is well known that entanglement is the manifestation of quantum correlations between two systems when they are in inseparable state. We consider the spin singlet state as an example of an entangled state.

$\mid \psi^{-}>_{0,0^{\prime}}=\frac{1}{\sqrt{2}}\left[\left|0>_{0^{\prime}}\right| 1>_{0}-\left|1>_{0^{\prime}}\right| 0>_{0}\right]$ Typically, the sen- der holds one member of the state of the pair of qubits while putting the other member at the near end of the AFM spin chain of length N. The spin chain is in the ground state. When the spin 0 starts to interact with the first spin of the chain then the Hamiltonian includes this additional interaction term $\left(I_{0^{\prime}} \otimes J \sigma_{0} \cdot \sigma_{1}\right)$, where $\sigma_{0}$ and $\sigma_{1}$ are the Pauli spin operators for the 0 and 1 sites respectively and $J$ is the exchange coupling. The initial state being $|\psi(0)>=| \psi^{-}>_{0,0^{\prime}} \otimes \mid \psi_{g}>$ where $\left|\psi_{g}\right\rangle$ is the ground state wave function of the AFM Hamiltonian and $|\psi(0)\rangle$ is the ground state wave function of the total Hamiltonian. This initial state starts to evolve and from that one can compute the density matrix and concurrence to measure the entanglement and purity of states. This is conventional wisdom of entanglement transport in the existing literature.

Motivations: But the goal of this letter is different: to solve the problem of entanglement transport orthogonally. Our main motivation is to interconnect the quantum many body physics and quantum information science. It is common practice in quantum many body physics to create a particle at any point in the system and study the dynamics of that particle to understand the physical behavior of the system. Therefore, we consider that one of the spin ( $\uparrow$ or $\downarrow$ ) of the singlet interacts with the spin chain and this spin itself transports through the 
chain medium due to the adiabatic variation of exchange couplings of the Hamiltonian, and reaches the other end of the chain.

We will explain the entanglement transport by calculating the entanglement current not by calculating concurrence and fidelity that reflect the orthogonality to solve this problem [11]. We achieve the perfect entanglement transport and also find a condition for blocking the entanglement transport even in the perfect pumping condition. These rigorous analytical solution and physical explanation are absent in all previous studies [7-22].

The other goal of this letter is to provide the correct analytical and physical explanation of entanglement hopping in alternate sites. It discusses why the entanglement transport in AFM spin chain outperforms the FM spin chain and also why the entanglement transport is better for a longer spin chain than for the shorter one.

Before we proceed further we would like to state the basic aspects of adiabatic pumping process (one of the elegant process of quantum many-body physics). An adiabatic parametric quantum pump is a device that generates a dc current by a cyclic variation of system parameters, the variation being slow enough that the system remains close to the ground state throughout the pumping cycle $[24,25]$. It is well known that when a quantum mechanical system evolves, it acquires a time dependent dynamical phase and time independent geometrical phase [26]. The geometrical phase depends on the geometry of the path in the parameter space. In the adiabatic entanglement pumping process, the locking potential well carries a spin of the singlet pairs. As the locking potential well slides through the adiabatic variation of system parameters, it induces a current $(I)$ in the system. In this study we calculate the current of this spin transport, which transports a spin from one end of the chain to the other and as a result of which entanglement is transported (because the spin $0^{\prime}$ and 0 are singlet and monogonus in nature) from one side to the other. This quantization is topologically protected against the other perturbation as long as the gap along the loop remains finite $[27,28]$.

\section{Model Hamiltonians, Quantum Field Theoretical Studies and Physical Interpretations}

Here we consider two different Hamiltonian: $H_{1}$ with modulated exchange coupling in $X Y$ plane and $\mathrm{H}_{2}$ for onsite magnetic field modulation. Hamiltonians of the systems are the following.

$$
H_{1}=-\sum_{n} J\left(1-(-1)^{n} \delta_{1}(t)\right)\left(S_{+}^{n} S_{-}^{n+1}+S_{+}^{n+1} S_{-}^{n}\right)
$$

$$
+\sum_{n} \Delta S_{z}^{n} S_{z}^{n+1}
$$

This model Hamiltonian has some experimental relevance [27]. The other model Hamiltonian with onsite magnetic field modulation is

$$
\begin{aligned}
H_{2}= & -\sum_{n} J\left(S_{x}^{n} S_{x}^{n+1}+S_{y}^{n} S_{y}^{n+1}\right)+\sum_{n} \Delta S_{z}^{n} S_{z}^{n+1} \\
& -\frac{1}{2} \sum_{n} B_{0}\left(1-(-1)^{n} \delta_{2}(t)\right) S_{z}^{n}
\end{aligned}
$$

Here we consider that the modulation is periodic over two lattice sites. We see that this model have essential ingredients to capture the adiabatic entanglement pumping.

Here, we would like to present the basic analytical methods to study the entanglement transport physics. In one dimensional quantum many body system, the Luttinger liquid (LL) theory based method is one of the most successful analytical methods to study the quantum systems. The analytical technique to implement LL picture is the Abelian bosonization method. At first, we express the spin Hamiltonians in terms of spinless fermion (annihilation and creation) operators. After that stage, we use the Abelian bosonization method to solve these Hamiltonians. One can express spin chain systems to a spinless fermion systems through the application of Jordan-Wigner transformation. In Jordan-Wigner transformation the relation between the spin and the electron creation and annihilation operators are

$$
\begin{aligned}
& S_{n}^{z}=\psi_{n}^{\dagger} \psi_{n}-1 / 2, \\
& S_{n}^{-}=\psi_{n} \exp \left[i \pi \sum_{j=-\infty}^{n-1} n_{j}\right], \\
& S_{n}^{+}=\psi_{n}^{\dagger} \exp \left[-i \pi \sum_{j=-\infty}^{n-1} n_{j}\right],
\end{aligned}
$$

[29], where $n_{j}=\psi_{j}^{\dagger} \psi_{j}$ is the fermion number at site $j$. Spin operators in terms of bosonic field are the following.

$$
\begin{aligned}
& S_{n}^{x}=\left[c_{2} \cos (2 \sqrt{\pi K} \phi)+(-1)^{n} c_{3}\right] \cos \left(\sqrt{\frac{\pi}{K}} \theta\right), \\
& S_{n}^{y}=-\left[c_{2} \cos (2 \sqrt{\pi K} \phi)+(-1)^{n} c_{3}\right] \sin \left(\sqrt{\frac{\pi}{K}} \theta\right), \\
& S_{n}^{z}=\sqrt{\frac{\pi}{K}} \partial_{x} \phi+(-1)^{n} c_{1} \cos (2 \sqrt{\pi K} \phi) .
\end{aligned}
$$

$K$ is the Luttinger liquid parameter, $c_{2}$ and $c_{3}$ are the constants. The fermionic fields,

$$
\psi_{r}(x)=\frac{U_{r}}{\sqrt{2 \pi \alpha}} \mathrm{e}^{-i(r \varphi(x)-\theta(x))}
$$


$r$ denotes the chirality of the fermionic fields, right (1) or left movers $(-1)$. The operators $U_{r}$ (Klein operators) preserve the anti-commutivity of the fermionic fields $\phi$ field corresponds to the quantum fluctuations (bosonic) of spin and $\theta$ is the dual field of $\phi$.

Using the standard machinery of continuum field theory [29], we finally get the bosonized Hamiltonians as

$$
\begin{gathered}
H_{1}=H_{0}+\frac{E_{J_{0}} \delta_{1}(t)}{2 \pi^{2} \alpha^{2}} \int \mathrm{d} x: \cos [2 \sqrt{K} \phi(x)] \\
+\frac{\Delta}{2 \pi^{2} \alpha^{2}} \int \mathrm{d} x: \cos [4 \sqrt{K} \phi(x)] \\
H_{2}=H_{0}+\frac{B_{0} \delta_{2}(t)}{2 \pi \alpha} \int \mathrm{d} x: \cos [2 \sqrt{K} \phi(x)] \\
-\frac{B_{0}}{2} \int \mathrm{d} x \partial_{x} \phi(x)+\frac{\Delta}{2 \pi^{2} \alpha^{2}} \int \mathrm{d} x: \cos [4 \sqrt{K} \phi(x)]
\end{gathered}
$$

$H_{0}$ is the gapless Tomonoga-Luttinger liquid part of the Hamiltonian.

We consider spin singlet as a reference entangled state. Therefore, we would like to explain the basic aspects of quantum entanglement pumping in terms of spin pumping physics of our model Hamiltonians. An adiabatic sliding motion of one dimensional potential, in gapped Fermi surface (insulating state), pumps an integer numbers of particle per cycle. In our case the transport of JordanWigner fermions (spinless fermions) is nothing but the transport of spin from one end of the chain to the other end because the number operator of spinless fermions is related to the z-component of spin density [30]. We will see that the non-zero $\delta_{1}(t)$ and $\delta_{2}(t)$ introduce the gap at around the Fermi point and the system is in the insulating state (Peierls insulator).

Here, we would like to discuss the physics of geometric phase related to our model Hamiltonians and its relation to the entanglement current. It is well known that the physical behavior of the system is identical at these two Fermi points. We would like to analyses these double degeneracy points following the seminal paper of Berry [26]. In our model Hamiltonian there are two adiabatic parameters $\delta_{1}(t)$ and $\delta_{2}(t)$. The Hamiltonian starts to evolve under the variation of these two adiabatic parameters. When the Hamiltonian returns to its original form after a time $T$, the total geometric phase acquired by the system is

$$
\gamma_{n}(T)=\frac{i}{2 \pi} \int_{C}\left\langle\psi_{n}\left|\nabla_{R}\right| \psi_{n}\right\rangle \mathrm{d} R,
$$

A line integral around a closed loop in two dimensional parameter space. Using Stokes theorem, one can write

$$
\gamma_{n}(T)=\frac{i}{2 \pi} \int \nabla_{R} \times\left\langle\psi_{n}\left|\nabla_{R}\right| \psi_{n}\right\rangle \mathrm{d} S
$$

The flux $\Phi$ through a closed surface $C$ is, $\Phi=\int B \cdot \mathrm{d} S$. Therefore, one can think of the Berry phase as flux of a magnetic field. Now we express,

and

$$
B_{n}(K 1)=\nabla_{K 1} \times A_{n}(K 1)
$$

$$
A_{n}(K 1)=\frac{i}{2 \pi}\left\langle n(K 1)\left|\nabla_{K 1}\right| n(K 1)\right\rangle,
$$

where $K 1=\left(k, \lambda_{1}(t), \lambda_{2}(t)\right), \quad \lambda_{1}(t)$ and $\lambda_{2}(t)$ are the real and imaginary part of the fourier transform of $\delta_{1}(t)$. Similarly for the Hamiltonian $H_{2}$, the adiabatic parameters will be different. Here $B_{n}$ and $A_{n}$ are the fictitious magnetic field (flux) and vector potential of the nth Bloch band respectively. The degenerate points behave as a magnetic monopole in the generalized momentum space $\left(K_{1}\right)$ [26], whose magnetic unit can be shown to be 1 analytically as $\int_{S 1} \mathrm{~d} S \cdot B_{ \pm}= \pm 1$ where positive and negative signs of the above equations are respectively for the conduction and valance band meeting at the degeneracy points $[26,27] . S_{1}$ represent an arbitrary closed surface which enclose the degeneracy point. In the adiabatic process the parameters $\delta_{1}(t)$ or $\delta_{2}(t)$ are changed along a loop $(\Gamma)$ enclosing the origin (minima of the system). We define the expression for spin current $(I)$ from the analysis of Berry phase. It is well known in the literature of adiabatic quantum pumping physics that two independent parameters are needed to achieve the adiabatic quantum pumping in a system [31]. Here one may consider these two parameters as the real and imaginary part of the fourier transform of a modulated coupling induced potential. When the shape of the potential will change in time, then it amounts to changing the phase and amplitude in time. We define the expression for spin current $(I)$ from the analysis of Berry phase. Then according to the original idea of quantum adiabatic particle transport [24,25, $27,28]$, the total number of spinless fermions $(I)$ which are transported from one side of this system to the other is equal to the total flux of the valance band, which penetrates the $2 \mathrm{D}$ closed sphere $\left(S_{2}\right)$ spanned by the $\Gamma$ and Brillioun zone [27].

$$
I=\int_{S_{2}} \mathrm{~d} S \cdot B_{+1}=1
$$

$B_{+1}$ is directly related with the Berry phase $\left(\gamma_{n}(T)\right)$ which is acquired by the system during the adiabatic variation of the exchange couplings during the time period of the adiabatic process. This quantization is topologically protected against the other perturbation as long as the gap along the loop remains finite [27,28]. Therefore, the adiabatic entanglement pumping is cons- 
tant over the arbitrarily long distance of the system. This result is in contrast with the existing results in the literature $[8,9,20]$. Therefore we have solved the problem of entanglement transport in terms of entanglement current but not in terms of conventional wisdom of the literature [8-22]. The authors of Ref. [23] have found that the entanglement decays exponentially after a certain distance.

Now we explain the quantum entanglement transfer for $H_{1}$. The second term of the Hamiltonian for NN exchange interaction has originated from the $x$ and $y$ component of exchange interaction. This term implies that infinitesimal variation of coupling in lattice sites and is sufficient to produce a gap around the Fermi points. So when $1 / 2<K<1$, only these time dependent exchange couplings are relevant and lock the phase operator at $\phi=0+\frac{n \pi}{\sqrt{K}}$. Now the locking potential slides adiabatically. The speed of the sliding potential is low enough such that the system stays in the same potential valley, i.e., there is no scope to jump onto the other potential valley. The system will acquire $2 \pi$ phase during one complete cycle of the adiabatic process. This expectation is easily verified when we notice the physical meaning of the phase operator $(\phi(x))$. Since the spatial derivative of the phase operator corresponds to the $z$-component of spin density, this phase operator is nothing but the minus of the spatial polarization of the $z$-component of spin, i.e., $P_{S^{z}}=-\frac{1}{N} \sum_{j=1}^{N} j S_{j}^{z}$. During the adiabatic process $\left\langle\phi_{t}\right\rangle$ changes monotonically and acquires $-2 \pi$ phase. In this process $P_{s}^{z}$ increases by 1 per cycle. We define it analytically as $[27,32]$

$$
\delta P_{s}^{z}=\int_{\Gamma} \mathrm{d} P_{s}^{z}=-\frac{1}{2 \pi} \int \mathrm{d} x \partial_{x}\langle\varphi(x)\rangle=1
$$

This physics always hold as far as the system is locked by the sliding potential and $\Delta<1$ [27]. The change of the spatial polarization by unity during a complete evaluation of adiabatic cycle implies the transport of entanglement across the system. The entanglement transport of this scenario can be generalized up to the value of $\Delta$ for which $K$ is greater than $1 / 2$. In this limit, z-component of the exchange interaction has no effect on the entanglement pumping of our system. But when $K<1 / 2$, then the interaction due to $\Delta$ becomes relevant and creates a gap in the excitation spectrum. This potential profile is static. Therefore, there is no scope to slide the potential and to get a adiabatic pumping across the system.

Similarly, for the Hamiltonian $\mathrm{H}_{2}$, the second term of the Hamiltonian produces the gap and the pumping process is the same as that of $H_{1}$. Therefore, we conclude that the modulations in plane exchange coupling and also for the modulations in the on site magnetic field yield the same adiabatic entanglement pumping. If we consider the unmodulated exchange coupling in our spin chain system, then there is no gap in the excitation spectrum. Therefore, there is no entanglement transport in our system. As we have been already described, in adiabatic quantum pumping process gap is absolutely necessary.

In this pumping process the most favorable states of the system are the antiferromagnetic configuration

$\mid 010101 \ldots>$ and $\mid 101010,,,>$ (0 stands for up spin and 1 stands for down spin). One may start from any antiferromagnetic states and transfer the spin of every site to the right by two sites to achieve the pumping. Therefore, our test spin which we introduce at the one end of the spin, hops to the right by two sites in every step. Thus when we study the entanglement transport between the spin $0^{\prime}$ and 0 , it is natural that the entanglement also is transported through every alternate sites. This is the first correct and complete analytical and physical explanation in the literature. The authors of Ref. $[7,22]$ have observe, the non analytical behavior of entanglement transport as a function of time. But in our study the entanglement current is constant and it is almost perfect entanglement pumping. In their case the spin chain has the spin rotational symmetry. When one member of an entangled pair of qubits is transmitted through such a channel, then the two qubits states evolve to a Werner state [33]. But our spin chain systems has no spin rotational invariant symmetry and the transport mechanism is also different.

Here, we would like to explain the difference of entanglement transport between the FM and AFM spin chain. It is mentioned in the literature but the complete physical explanation is not upto the mark [7-18,22]. Here we consider the AFM/FM spin chain with static exchange coupling to use the result of Bethe ansatz calculations. As we know that entanglement is a quantum mechanical property, Schrodinger singled out many decades ago as the characteristic of quantum mechanics [34] and that has been studied extensively in connection with Bell's inequality [35]. In FM ground state, there is no difference between the classical and quantum mechanical ground state and the low lying excitations are spin-1 magnons. The AFM ground state has a complex structure specified by the Bethe-ansatz solution. There are no similarities between classical and quantum mechanical ground state and first excited state of the AFM chain and as a result of the quantum mechanical property of the system, the entanglement manifests prominently in the AFM spin chain. This is the only clear reason why AFM outper- 
forms the FM spin chain as far as entanglement related properties are concerned. This correct physical analysis was absent in all previous studies.

Here we discuss possible sources of imperfections in the entanglement pumping process of our system. The non-adiabatic contributions leave the system in an unknown superposition of states after the full cycle. Also, the appearance of Landau-Zener transition in the pumping system should be negligible so that the system is in the ground state. This condition limits the pumping rate of entanglement by the mathematical relation $\frac{h}{\tau} \ll J$. However, even then the entanglement pumping is not perfect due to the non vanishing $\frac{J}{\Delta}$. Our effort also should take the elimination of entanglement pumping in the wrong directions. The residual exchange coupling may lead to a different spin state. An entangled spin transported through a correct exchange coupling modulation with probability $P$ and through the residual exchange coupling with the probability $Q=1-P$. Therefore, the pumping error in each site is $\frac{Q}{P}$. During this analysis we assume that $P>Q$. Our system consists of $N$ sites. So the probability of correct entanglement transport is $\sim P^{N / 2}$ and wrong entanglement transport is $\sim Q^{N / 2}$. The total pumping error, $\left(\frac{Q}{P}\right)^{N / 2}$, decreases with the number of sites in nanoscale spin chain. Therefore, for the spin chain system entanglement transport is better for larger length compared to the smaller length with the same exchange couplings. This finding is in contrast with the previous findings.

\section{Conclusions}

We have presented the theoretical explanation of perfect adiabatic entanglement pumping for our model Hamiltonians. We have solved this problem orthogonally. We have defined and calculated the entanglement current for the first time in the literature. We have also found a subclass for blocking of entanglement transport, even in the perfect pumping condition. We have explained many physical findings of entanglement transport, such as hopping of entanglement in alternate sites and the fact that entanglement transport in AFM spin chain outperforms the FM spin chain. These facts were subject of curiosity before our study. We have explained analytically and physically the reasons why the entanglement transport is better for the larger length scale compared to the smaller one. Our rigorous analytical solutions inter- connect quantum many body physics and quantum information science.

The author would like to thank the Center for Condensed Matter Theory of IISc for extended facility. The author would like to give special thanks to Prof. Arnab Roychoudhuri for constant encourgement to produce better quality of work. Finally the author would like to thank Prof. N. Behera, Prof. R. Srikanth, Dr. T. Tulsi and Prof. Indrani Bose.

\section{References}

[1] A. J. Skineer, et al., "Hydrogenic Spin Quantum Computing in Silicon: A Digital Approach," Physical Review Letters, Vol. 90, No. 8, 2003, pp. 087901-087904. doi:10.1103/PhysRevLett.90.087901

[2] A. Zeilinger, "Experiment and the Foundation of Quantum Physics," Reviews of Modern Physics, Vol. 71, No. 2, 1999, pp. S288-S297.

[3] A. Rauschenbeutel, et al., "Step by Step Engineered Multiparticle," Science, Vol. 288, No. 5473, 2000, pp. 2024 2029. doi:10.1126/science.288.5473.2024

[4] C. A. Sackett, et al., "Experimental Entanglement of Four Particle," Nature, Vol. 404, No. 6775, 2000, pp. 256-258. doi:10.1038/35005011

[5] M. Bayer, et al., "Coupling and Entanglement of Quantum States in Quantum Dot Molecules," Science, Vol. 291, No. 5503, 2001, pp. 451-453. doi:10.1126/science.291.5503.451

[6] F. Plastina, R. Fazio and G. M. Palma, "Macroscopic Entanglement in Josephson Nanocircuits," Physical Review B, Vol. 64, No. 11, 2001, pp. 113306-113310. doi:10.1103/PhysRevB.64.113306

[7] S. Bose, "Quantum Computation through Unmodulated Spin Chain," Physical Review Letters, Vol. 91, No. 20, 2003, pp. 207901-207904. doi:10.1103/PhysRevLett.91.207901

[8] V. Subrahmanyam, "Entanglement Dynamics and Quantum State Transport in Spin Chain,” Physical Review A, Vol. 69, No. 3, 2004, pp. 034304-034307.

[9] A. Kay, "Unifying Quantum State Transfer and State Amplification," Physical Review Letters, Vol. 98, No. 1, 2007, pp. 010501-010504. doi:10.1103/PhysRevLett.98.010501

[10] T. J. Osborne and N. Linden, "Propagation of Quantum Information through a Spin System," Physical Review A, Vol. 69, No. 5, 2004, pp. 052315-052320. doi:10.1103/PhysRevA.69.052315

[11] L. Amico, et al., "Scaling of Entanglement Close to a Quantum Phase Transition," Nature, Vol. 416, No. 6881, 2002, pp. 608-610. doi:10.1038/416608a

[12] A. Bayat and V. Karimipour, "Thermal Effect on Quantum Communication through Spin Chain," Physical Review A, Vol. 71, No. 4, 2005, pp. 042330-042336. doi:10.1103/PhysRevA.71.042330 
[13] L. C. Venuti, et al., "Long-Distance Entanglement in Spin System," Physical Review Letters, Vol. 96, No. 24, 2006, pp. 247206-247209. doi:10.1103/PhysRevLett.96.247206

[14] O. Romero-Isart, K. Eckert and A. Sanpera, "Quantum State Transfer in Spin-1 Chain," Physical Review A, Vol. 75, No. 5, 2007, pp. 050303-050306. doi:10.1103/PhysRevA.75.050303

[15] K. Eckert, O. Romero-Isart and A. Sanpera, "Efficient Quantum State Transfer in Spin Chain via Adiabatic Passage," New Journal of Physics, Vol. 9, No. 5, 2007, pp. 155-173. doi:10.1088/1367-2630/9/5/155

[16] V. Srinivasa, J. Levy and C. S. Hellberg, "Flying Spin Qubit," Exchange Organizational Behavior Teaching Journal, 2006, pp. 1-13.

[17] M. J. Hartmann, et al., "Excitations and Entanglement Transfer versus Spectral Gap," New Journal of Physics, Vol. 8, No. 6, 2006, pp. 94-108. doi:10.1088/1367-2630/8/6/094

[18] L. Amico, et al., "Dynamics of Entanglement in OneDimensional Spin Systems," Physical Review A, Vol. 69, No. 2, 2004, pp. 022304-022327. doi:10.1103/PhysRevA.69.022304

[19] C. Di Franco, et al., "Perfect State Transfer of Spin Chain without State Initialization," Physical Review Letters, Vol. 101, No. 23, 2008, pp. 230502-230503. doi:10.1103/PhysRevLett.101.230502

[20] F. Plastina, et al., "Local Control of Entanglement in Spin Chain," Physical Review Letters, Vol. 99, No. 17, 2007, pp. 177210-177213. doi:10.1103/PhysRevLett.99.177210

[21] M. Horodecki, et al., "General Teleporation Channel, Singlet Fraction and Quasidistillation," Physical Review A, Vol. 60, No. 3, 1999, pp. 1888-1898.

[22] A. Bayat and S. Bose, "Information Transferring Ability of the Different Phases of a Finite XXZ Spin Chain," Physical Review A, Vol. 81, No. 1, 2008, pp. 1-11.

[23] C. F. Hirjibehedin, et al., "Spin Coupling in Engineered Atomic Structures," Science, Vol. 312, No. 5776, 2006, pp. 1021-1025. doi:10.1126/science. 1125398

[24] D. J. Thouless, "Quantization of Particle Transport," Physical Review B, Vol. 27, No. 10, 1983, pp. 6083-6087. doi:10.1103/PhysRevB.27.6083
[25] Q. Niu and D. J. Thouless, "Quantized Adiabatic Charge Transport in the Presence of Substrate Disorder and Many-Body Interaction," Journal of Physics A: Mathematical and General, Vol. 17, No. 12, 1984, pp. 2453 2462. doi:10.1088/0305-4470/17/12/016

[26] M. V. Berry, "Quantal Phase Factor Accompaying Adiabatic Charges," Proceedings of the Royal Society A, Vol. 392, No. 1802, 1984, pp. 45-57. doi:10.1098/rspa.1984.0023

[27] R. Shindou, "Quantum Spin Pump in S = 1/2 Antiferromagnetic Chain Holonomy of Phase Operators in SineGordon Theory," Journal of the Physics Society Japan, Vol. 74, No. 4, 2005, pp. 1214-1223. doi:10.1143/JPSJ.74.1214

[28] J. E. Avron, A. Raveh and B. Zur, "Adiabatic Quantum Transport in Multiple Connected System," Reviews of Modern Physics, Vol. 60, No. 4, 1988, pp. 873-915. doi:10.1103/RevModPhys.60.873

[29] T. Giamarchi, "Quantum Physics in One Dimension," Clarendon Press, London, 2004.

[30] $S_{n}^{z}=\frac{1}{2 \pi} \partial_{x} \phi\left(x_{n}\right)-\frac{(-1)^{n}}{\pi \alpha} \sin \left(\phi\left(x_{n}\right)\right) \cdot \phi$ field corresponds to the quantum fluctuations (boson) of spin.

[31] P. Sharma and C. Chamon, "Quantum Pump for Spin and Charge Transport in a Luttinger Liquid," Physical Review Letters, Vol. 87, No. 9, 2001, pp. 96401-96405. doi:10.1103/PhysRevLett.87.096401

[32] S. Sarkar and C. D. Hu, "Quantum Spin Pumping at a Fractionally Quantized Magnetization State for a System with Competing Exchange Interactions," Physical Review B, Vol. 77, No. 6, 2008, pp. 064413-064418. doi:10.1103/PhysRevB.77.064413

[33] C. H. Bennett, et al., "Mixed State Entanglement and Quantum Error Correction," Physical Review A, Vol. 54, No. 5, 1996, pp. 3824-3851. doi:10.1103/PhysRevA.54.3824

[34] E. Schrodinger, "Discussion of Probability Relation between Separated System," Mathematical Proceedings of the Cambridge Philosophical Society, Vol. 31, No. 4, 1935, pp. 555-563.

[35] J. S. Bell, "On the Einstein-Podolsky-Rosen Paradox," Physics, Vol. 1, No. 3, 1964, pp. 195-202. 